\title{
RIMAPS Studies on Structured Copper Foils
}

\author{
M Flores Zavala ${ }^{1}$, P Löthman ${ }^{2}$ and EA Favret ${ }^{3,4}$ \\ ${ }^{1}$ Instituto Sabato, UNSAM - CNEA, Buenos Aires, Argentina \\ ${ }^{2}$ KIST Europe Forschungsgesellschaft GmbH, Saarbrücken, Germany \\ ${ }^{3}$ Instituto de Suelos, CIRN, INTA. Hurlingham. Argentina \\ ${ }^{4}$ CONICET. Buenos Aires. Argentina
}

It is known that RIMAPS (Rotated Image with Maximum Average Power Spectrum) technique allows finding the main directions of any surface topography and describing it by simple geometrical figures [1]. The maxima of the RIMAPS spectrum indicate main directions and the arrangement of the elements that constitute the surface. RIMAPS has previously been used in several fields, such as materials science and biology [2] [3]. In this opportunity, the objective was to apply RIMAPS to analyze the micro-nanostructured surface of copper foils which were manufactured via an electrolytic plating route. A high purity copper base foil was electroplated using a specific electrolyte bath. A defined ultraflat surface structure in combination with given mechanical properties was obtained. Electroplating an anti-tarnishing layer (sample A, see figure 1) or a roughening treatment with anti-tarnishing layer (sample B, see figure 2) on the smooth base foil give a specific micro-nanotopography that could be characterized and differentiated by RIMAPS. The samples were observed using a scanning electron microscope (FEI Quanta 250). The surface elements of both samples differ in size. The RIMAPS spectra of samples A and B show many maxima, indicating several directions of the surface topography (see figure 3). The two spectra are quite similar. This result is not strange because the basic manufactured process is the same. For a better determination of the main directions (main maxima of the RIMAPS spectrum), a smoothing of the RIMAPS spectra based on Fast Fourier Transform was used (see figure 4). From these spectra it is possible to say about the topography of the samples that sample A has approximately 11 directions and sample $\mathrm{B}$ between 12 and 13 directions. If sample $\mathrm{A}$ is observed with a higher magnification surface elements like stars can be detected (see figure 5). Therefore we propose a model based on the form of a "star" for describing the main directions and arrangement of the surface elements of these copper foils (see figure 6). The number of lines that forms the star is the number of maxima of the smoothed RIMAPS spectrum in a rotation of $360^{\circ}$. The star representing the topography has 22 lines for sample A and 24 or 26 lines for sample B. Nevertheless other extra studies need to be done to validate this model. Nowadays RIMAPS spectra of star drawings with different number of lines are being calculated. Other parameters of the spectrum are also being considered, such as the integral of the curve which increases as the number of lines of the star increases. In conclusion, $a$ priori RIMAPS analysis seems to be a tool for developing models of geometrical figures that describes, in a simple manner, the micro-nanotopography of the surface. In the present case, a model based on stars could be appropriated [4].

\section{References}

[1] NO Fuentes and EA Favret, Journal of Microscopy 206 (2002), p. 72-83.

[2] SM Romero et al, Microscopy and Microanalysis 20 (Suppl 3) (2014), p. 1340-1341.

[3] EA Favret and P. Löthman, Microscopy and Analysis 21 (1) (2007), p. 7-9.

[4] The authors acknowledge the technicians of the microscopy laboratory of CICVyA, INTA as well as Laurence Vast at Circuit Foil Luxemburg for providing copper sheets. 


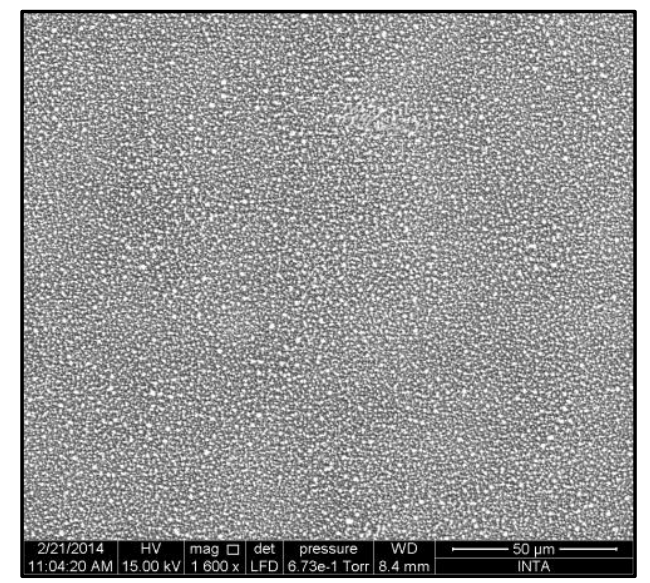

Figure 1. SEM micrograph of sample A

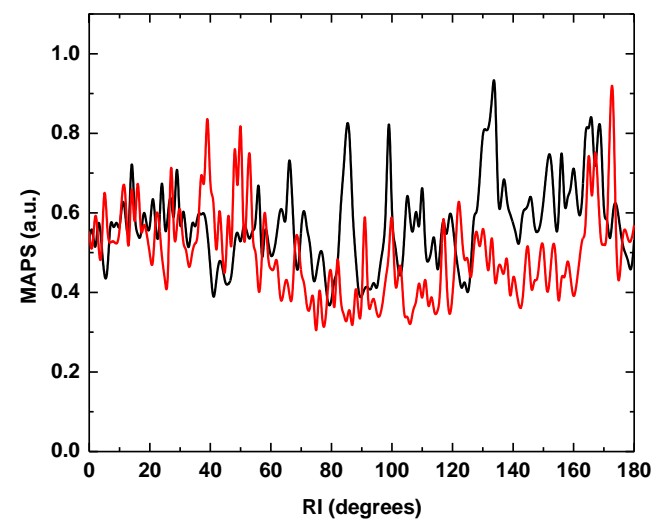

Figure 3. RIMAPS spectra of figure 1 (red line) and figure 2 (black line)

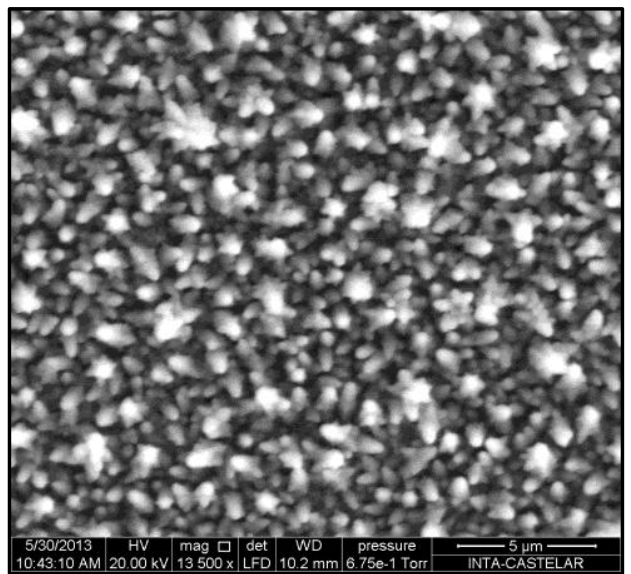

Figure 5. SEM micrograph of sample A with a higher magnification

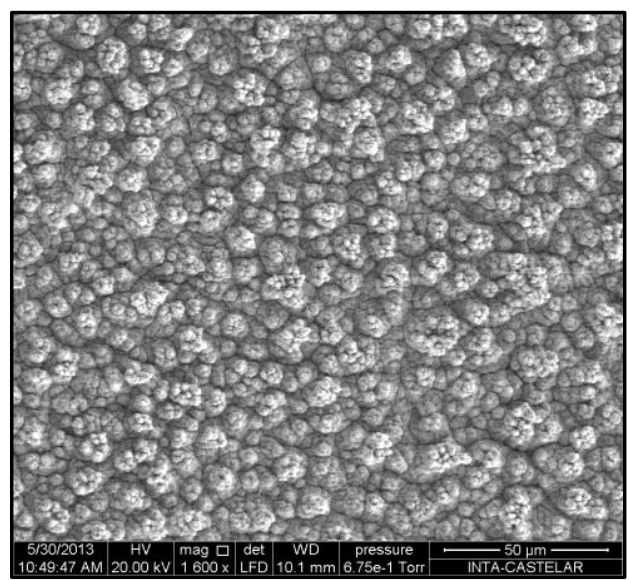

Figure 2. SEM micrograph of sample B

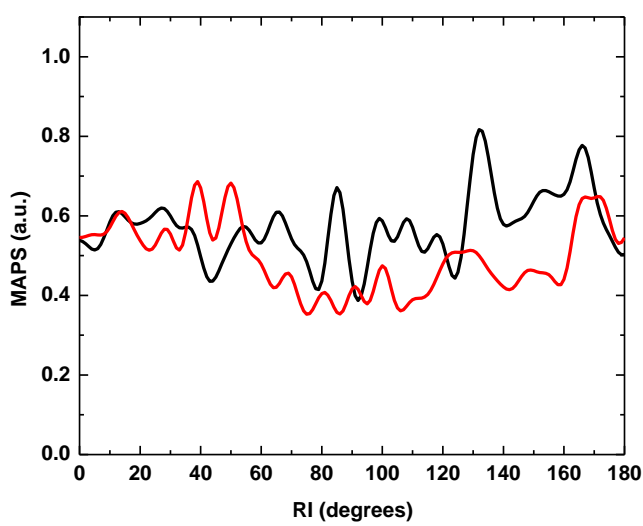

Figure 4. FFT smoothing (5 points) of RIMAPS spectra of figure 3

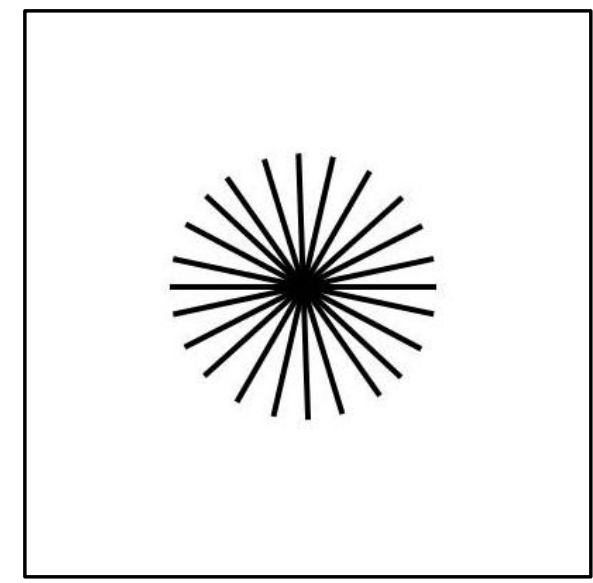

Figure 6. Sketch of the star model 